\title{
Research on Energy-stored Programmable 5.0 kA Surge Current Testing Equipment
}

\author{
Dai Juan and Zhao Zhihong \\ School of Energy and Electronical Engineering \\ Nanjing Institute of Industry Technology, China, 210023 \\ Daij@niit.edu.cn, Zhaozh@niit.edu.cn
}

\begin{abstract}
Surge current test data have been taken on as one important parameter among the process of the production and application of rectifier diode. According to the National Rectifying Diode Surge Current Testing Standards, surge current is usually generated from transformer and simultaneously tested by some testing special instruments, i.e. oscilloscopes. However, this kind of test can cause problems, such as extra high cost required, surge current waveforms distorted, and great influence made upon power grid fluctuations while high current tested. This paper addresses an efficient method to tackle those problems mentioned above. In this method, surge currents are generated by stored charges, then a microcomputer is adopted to control its surge waveform and the real time data are tested and displayed, such as the currents and voltages through the rectifier tube. Such technique can easily eliminate the effects of defects resulted from those surge current generated by transformer. Furthermore, it's already proved to be much more accurate, controllable in surge time and peak. Compared with the method of surge current generated by transformer, such energystored method also saves more energy and occupies small volumes of space. Recently, instruments developed with this method have been applied in production testing systems in a number of related enterprises, and yield to a favorite effect.
\end{abstract}

Keywords: surge current, 5.0 kA, stored charge, microcomputer

\section{Introduction}

With the increasingly fast development of power electronic technology and the rapidly increasing usage of various kinds of electrical devices, much more demand for making a great improvement on the power quality of AC sources has been of a great importance for decades. So many researches conducted on all of the devices or components used to improve the quality in the power department should be considered and discussed in detail. Among them, rectifier diode, a semiconductor device with a $\mathrm{PN}$, is mainly used to change AC (alternating current) into DC (direct current) in small or medium-to-high scale power applications. By definition, surge current refers to the peak current flowing into the power equipment in the moment of switching power on. Theoretically speaking, a desired single-phase rectifier diode may be directly loaded into the individual applications without any tests as it definitely has a consistent resistive load to the objective AC-DC power system. But in actual cases, it has to be tested through various kinds of professional testing equipments. Such surge current testing process is one of the most important aspects among all of the measures to keep the whole system safe and stable running. Requirement for surge current test is mainly to apply such wave current into rectifier diode which caused by abnormal circuit circumstances (i.e., fault). 
Unfortunately, its temperature may meet or exceed the maximum rated value and damage or fire disaster will occur when the PN junction is applied by such kind of current [1]. Therefore, surge current parameters testing (SCPT) is of great importance for anti-abnormal feature of rectifier diodes, which has a direct impact on standards used for production by manufacturers.

Surge current testing (SCT) can be considered not only as measurement process, but also as surge current generation. There is an explanation of Chinese National standard GB/40231997 related to surge current rated value and characteristic terms. It says that forward (nonrepetitive) surge current IFSM is a forward current impulse which is short and has the specified waveform, i.e., a sine wave. In such standard, what is used to generate surge current is mainly focused on transforming output method. This method is direct, simple and easy to operate. However, there are some shortages such as high cost, big power consumption, easy to be influenced by power grid, and difficult to control a number of indicators when high level surge current is generated to meet the demand. Recently, a large number of researches have been lunched and conducted on efficiently and accurately testing such surge current. In order to solve those problems caused by these heavy currents, an energy-stored method is adopted to generate surge current. By comparison, the result shows that the capability and validity of the addressed method is tested and approved in an experimental manner and its effectiveness of power consumption and cost performance is also much better than that of surge current testing conventional methods.

The rest of this paper is organized as follows. Surge current-generating and -testing system of this paper are described in Section 2. It mainly includes three aspects. Firstly, an energystored SCT system structure is briefly introduced in Section 2.1. Secondly, the authors will discuss the charge storage of 5.0kA surge current in Section 2.2. How to control the precision of the sine wave current with peak value of $5.0 \mathrm{kA}$ will be discussed in detail in Section 2.3. The comparison we will discuss in greater detail later between energy-stored surge current method and transformer surge method is elaborated in Section 3. Such comparison will be focused on four necessary items, i.e., the minimum period, the surge waveform standards, the power consumption and cost, and the others. The conclusion of the system and the focus of further research will be presented and discussed in Section 4. We then analyze the limitations of such addressed approaches and also highlight the contributions of our algorithms.

\section{Surge Current-generating and -testing System}

In the national standard GB4023-1997, i.e., Part 2 rectifier diodes, in discrete devices and integrated circuits of semiconductor devices, surge current is defined as a short forward current impulse with a certain specified sine wave. According to the requirement, the testing circuit of Non-Repetitive Peak Forward current IFSM only works within a forward semi-period and its turn-on angle is increased approximately to 180 degrees [1]. It is generally suggested that forward (surge) semi-period current should be provided by low-voltage high-current transformers.

For low-power rectifier diodes, the required forward surge current may be provided by general transformers. However, if transformers are required to provide a $5.0 \mathrm{kA}$ current, due to large current, the coiled wire diameter of transformers may reach higher values according to the fact that current value is linearly proportional to the coiled wire diameter. Meanwhile, the more thermal energy is generated as the current rises up, with the result that the power consumption is increased to a higher level. Its volume can be also increased correspondingly when larger coiled wires are adopted in a transformer [2]. It is totally inconvenient for those SCT enterprises' applications. 


\subsection{Energy-stored SCT system structure}

The energy-stored SCT system consists of the following three main modules, i.e. chargeable energy storage module, surge discharge module, data acquisition and display module, as shown in Figure 1.

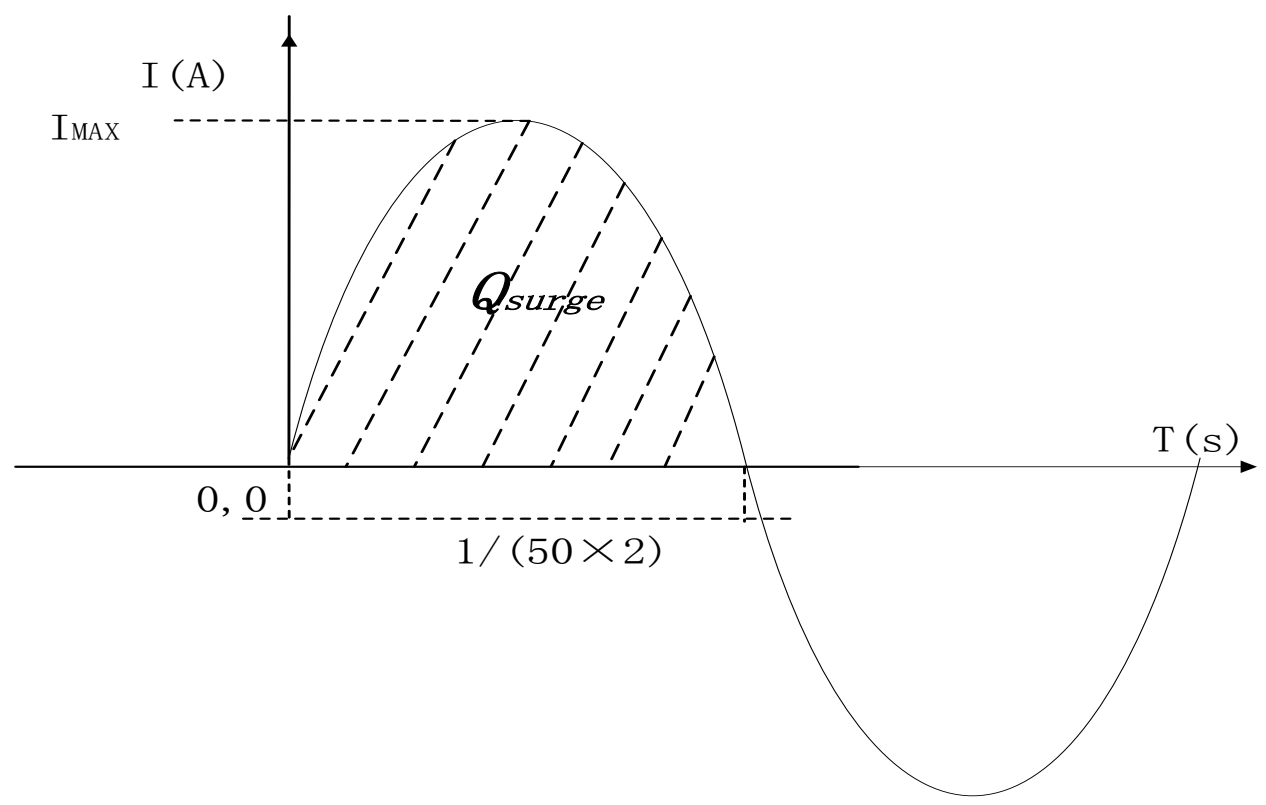

Figure 1. Energy-stored SCT system structure

Chargeable energy storage module: This module generally consists of some required charging circuits and other necessary energy storage circuits. Its main function is to transform power frequency electric energy into single-direction signal energy which will be correspondingly stored in those storage devices. Large capacitors other than batteries may be selected as these storage devices due to the case that the later can not release large currents instantly.

Surge discharge module: Such discharge module mainly includes two components, i.e. the surge driven control and the discharge units. It is used to generate the expected standard surge current within a specified time period. Its wave shape and peak will approximately meet the national standards. Such module may be constituted by a surge signal generating circuit including microcomputer and DAC (Digital to Analog Converter) module, or by discharge tube circuits consisting of NPN Darlington transistors [3].

Data acquisition and display module: Data acquisition and display module consists of signal acquisition, conversion and display units. It is mainly used to acquire the instant surge current values and then display them on. It consists of a microcomputer, ADC (Analog to Digital Converter) and LCD screen circuit.

The operation process of such system is now briefly discussed. After the transformer converts $220 \mathrm{~V}$ voltage into the expected lower one, it will flow through a rectifier diode and a unidirectional fluctuating current is shaped. Thus, the storage capacitor is then charged easily. When the electric switch is turned on by microcomputer, the current may flow through the charging current-limiting resistor and then the energy-stored capacitor will be charged instantly. Meanwhile, the voltage of this energy-stored capacitor is sampled. If such voltage sampled rise up to the predetermined value, the charging electric switch is then turned off and 
simultaneously turn on the relay. Therefore, the discharging circuit starts to open and waits for the SCT control signal.

When the microcomputer receives SCT starting signal, the program will begin to run and simulate the digital signal used to approximately describe a sine wave. Such digital signal then can be transmitted to the DAC module with the result that we may achieve the analog current signal in relation to the sine wave. This analog current signal is subsequently transmitted to the base of the discharging tube consisted of the Darlington transistor array. Under the control of base, the electric stored in the storage capacitor will be released in the specific time according to sine wave, and the current is formed. The sampling resistor voltage is sampled and measured while the current is released by the discharging tube controlled by the microcomputer. After that, the surge current peak value may be calculated and displayed.

Obviously, there exist two problems which need to be discussed in the process, i.e. how to ensure the amount of storage charge in order to make it possible to release sine wave surge current with the peak value of $5.0 \mathrm{kA}$, and how to ensure the sine wave peak accuracy of 5.0 kA.

\subsection{Charge storage of 5.0kA surge current}

We should firstly figure out Surge of 5.0kA surge current. As the formula $Q=I \times t$ is known, it takes seconds for a $50 \mathrm{~Hz}$ sine wave in a semi-period, and the surge current with

peak current $\mathrm{I}_{\mathrm{MAX}}=5.0 \mathrm{kA}$ may be shown in Figure 2 .

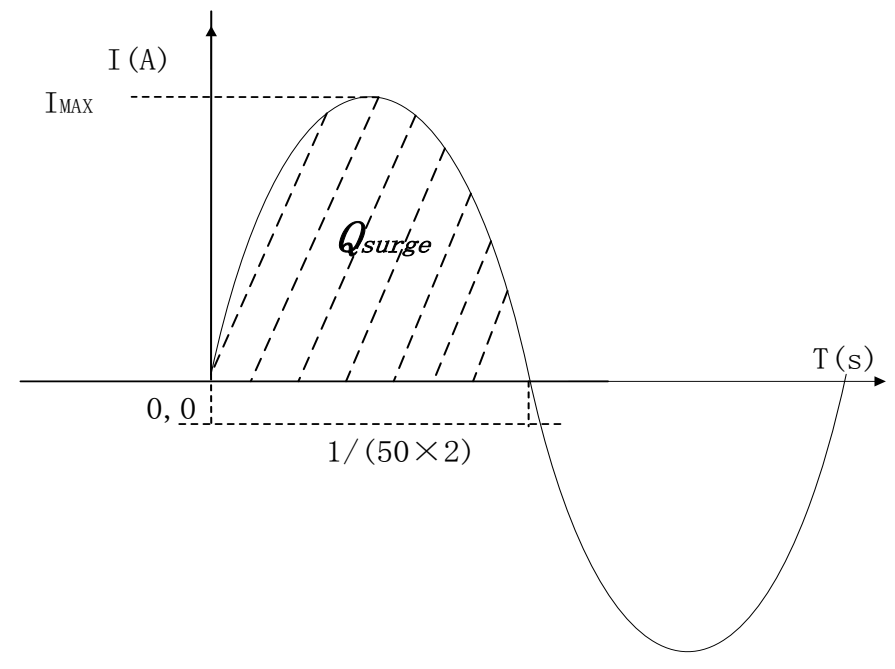

\section{Figure 2. A complete curve diagram for surge current}

In Figure 2, the charge flow will be blocked during the negative half cycle or converted to flow during the positive half cycle due to the unidirectional attribute of a diode. Therefore, some per- formation should be employed for the surge current wave during a half cycle. Tthe shaded area is used to represent QSurge, which meets a sine wave integral formula, i.e.,

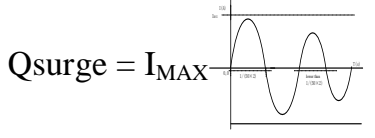


Put the current peak $\mathrm{I}_{\mathrm{MAX}}=5000$ and $\stackrel{\square}{-}$ into (1), and we will get

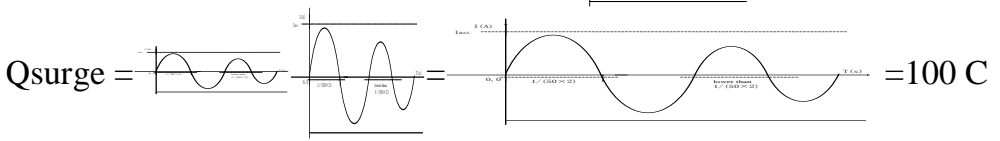

If capacitor $\mathrm{C}$ is used to store Qsurge, a certain potential can be reflected on it, shown as

$\mathrm{V}_{\text {Potential }}=$ Qsurge $/ \mathrm{C}$

By Equation (3), we may calculate the corresponding voltage $\mathrm{VC}$ on the capacitor. Measuring such voltage, we will find out the amount of electric charge inside of the capacitor.

In Figure 1 mentioned above, the discharge tube saturation voltage drop is $1.5 \mathrm{~V}$, and the rectifier diode voltage drop is $0.3-0.7 \mathrm{~V}$ (here we select $0.7 \mathrm{~V}$ ). Although the capacitor continuously varies discharge, the amount of charge cannot be reduced to the bottom and some of the rest charge are needed to meet its basis voltage $\mathrm{V}_{\text {Base }}=1.5+0.7=2.2 \mathrm{~V}$. Therefore, the amount of charging charge is defined by $\mathrm{Q}_{\text {Charging }}=\mathrm{Q}_{\text {surge }}+\mathrm{Q}_{\text {Base }}$.

When the capacitor $2.5 \mathrm{~F}$ is selected, the voltage on it is may be calculated by

$$
\mathrm{V}_{\mathrm{C}}=\left(\mathrm{Q}_{\text {surge }}+\mathrm{Q}_{\text {Base }}\right) / \mathrm{C}=100 / 2.5+2.2 \approx 43(\mathrm{~V})
$$

$\mathrm{V}_{\mathrm{C}}$ in Equation (4) is a minimum voltage value which can ensure the capacitor releasing $5.0 \mathrm{kA}$ surge current. Now, let's talk about the second voltage $\mathrm{V}_{\text {Second }}$ of the transformer used to charge the capacitor. Assuming that the peak voltage is equal to $45 \mathrm{~V}$ (needs to be greater than $43 \mathrm{~V}$ ) after being rectified and a full-wave rectifier is adopted in the charging circuit, we will get

$$
\mathrm{V}_{\text {Second }}=\mathrm{V}_{\mathrm{C}} / \operatorname{sqrt}(2) \approx 32 \mathrm{~V} \text {. }
$$

On the other hand, due to the charging current-limiting resistor existing in the charging circuit, we should consider its voltage drop (varies as the charging current changes). Thus, transformers with the primary and secondary voltages of $220 \mathrm{~V}$ and $40 \mathrm{~V}$ can be selected and fully meet our requirements.

When selected a charging current-limiting resistor of $5 \mathrm{Ohm}$, the charging current maximum in the system is calculated as $40 \times \operatorname{sqrt}(2) / 5 \approx 11 \mathrm{~A}$. The maximum power of the required transformer may be easily defined by

$\mathrm{Ps}=\mathrm{U} \times \mathrm{I}=11 \times 40 \times \operatorname{sqrt}(2) \approx 622 \mathrm{~W}$.

However, if the transformer is directly used, the required maximum power is

$\mathrm{PD}=\mathrm{U} \times \mathrm{I}=0.7 \times 5000=3500 \mathrm{~W}$.

Obviously, Ps is much smaller than PD.

\subsection{Controlling precision of the sine wave current with peak value of $5.0 \mathrm{kA}$}

Now, we attend to simulate a sine wave by using a program segment, shown as

$$
\text { for }(\mathrm{x}=0 ; \mathrm{x}<\pi ; \mathrm{x}+=0.01) \quad \mathrm{Px}=2^{8} \sin (\mathrm{x}) \text {. }
$$

Here, $\mathrm{x}$ represents time, and Px represents the signal of current amplitude data.

We adopt a digital signal to simulate sine-wave. Through digital-to-analog conversion, a minor error will exist. According to a formula for the slope of a curve [4], when $5.0 \mathrm{kA}$ surge 
current generated, the maximum error point will be located where the first order derivative value of sine wave reaches maximum, i.e., $\cos 0$. Its maximum absolute error is $5000 \times \mid \cos 0$ $\cos 0.01 \mid=0.25 \mathrm{~A}$, and its relative error is $(0.25 / 5000) \times 100 \%=0.005 \%$. Obviously, it is easy to meet the requirements under the normal cases.

If the accuracy is increased to a higher level, the progressive values for $\mathrm{x}$ should be decreased smaller, e.g., $\mathrm{x}+=0.001$. Thus, its absolute error is greatly reduced to $5000 \times \mid \cos 0$ $\cos 0.01 \mid=0.000005 \mathrm{~A}$. Only one requirement needs to be met, the speed of microcomputer should be properly enhanced in order that the loop for $(\mathrm{x}=0 ; \mathrm{x}<\pi ; \mathrm{x}+=0.001)$ is capable of being executed 1000 times in $10 \mathrm{~ms}$, i.e., the instruction cycle cannot be higher than 2 us.

\section{Comparison between Energy-stored Surge Current Method and Transformer Surge Method}

There are about four aspects to be compared between our method (i.e., energy-stored surge current) and the transformer surge method, discussed in detail as follows.

(1) Comparison on the minimum period

The surge minimum period includes two components, i.e.,

TSurge period $=$ tCharging time $+\mathrm{tDischarging}$ test time

Now, we may discuss the calculation for energy-stored charging time. Firstly, we assume that $\mathrm{Q}$ is the electric charge of the capacitor at time $\mathrm{t}$ with the corresponding parameters such as $\mathrm{V}$ (voltage), $\mathrm{E}$ (charging power supply), $\mathrm{R}$ (charging circuit resistor), and I (charging current). According to the circuit voltage formula, we'll get

$\mathrm{E}-\mathrm{V}=\mathrm{I} \times \mathrm{R}$

Now we enter

$\mathrm{V}=\mathrm{Q} / \mathrm{C}$

and

$\mathrm{I}=\mathrm{dQ} / \mathrm{dt}$

into Eq.(10), and we will get

(13)
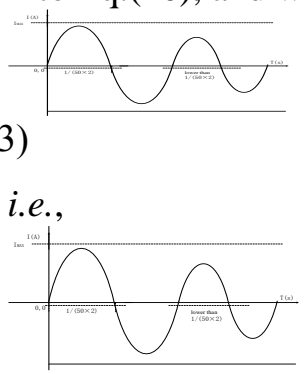

Then, we may calculate the indefinite integrals for both sides of Eq.(14). Combining with the initial conditions $\mathrm{t}=0$, and $\mathrm{Q}=0$, we'll get

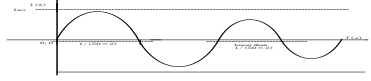

Equation (15) is what we try to use to represent the relationship between charge on the capacitor and time varying. Assuming that the voltage on the capacitor is Vt and the basic 
voltage is V0 at an instantaneous time t, Eq. (16) can be arrived when combined with Eq. (3) and. (4).

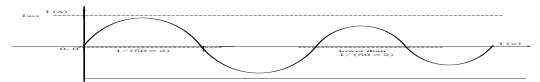

In Eq. (16), we define $\mathrm{V} 0=0$ as it is small and close to zero. Charging power supply (with voltage of $\mathrm{E}$ ) will charge the capacitor $\mathrm{C}$ through resistor $\mathrm{R}$. Therefore, the voltage on the capacitor at an instantaneous time $t$ may be calculated as

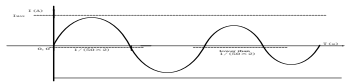

After Eq. (17) transformed, the log transformation is adopted on its both sides and Eq. (18) will be arrived, shown as

$\mathrm{t}=\mathrm{R} \times \mathrm{C} \times \mathrm{Ln}(\mathrm{E} /(\mathrm{E}-\mathrm{Vt}))$.

From Eq. (18), given Vt on the capacitor, $t$ can be calculated according to the constants $E$, $\mathrm{R}$ and $\mathrm{C}$. For example, the required time $\mathrm{t}$ will be approximately equal to $35 \mathrm{~s}$ if $\mathrm{R}=5 \Omega$, $\mathrm{C}=2.5 \mathrm{~F}$ and $\mathrm{V}_{\mathrm{t}}=45 \mathrm{~V}$.

The energy-stored surge current testing time will be reduced lower than 50s when taking some other factors into consideration, such as discharging time, sample time, data analysis time, and display time.

Obviously, the minimum period of energy-stored surge current is mainly restricted by the charging time. If a shorter surge current period is required, there exist no effective methods other than reducing the resistor and meanwhile increasing the powers of transformer and the resistor.

However, the transformer surge current is generally generated directly by transformer without any delay. Its minimum period is mainly affected by discharging time, sampling time and display time. Generally, it only takes 1 s to complete the mission.

(2) Comparison on the surge waveform standards

When using transformer to produce surge current, it will more or less depend on the grid. However, the secondary order waveform may be deformed to some extent when the grid is affected by the primary order signal. Meanwhile, its peak will vary randomly and can not be controlled with the result that the accuracy and repetition of parameters' testing will be heavily affected. In contrast, the waveform and peak can be easily controlled and the accuracy and repetition of parameters' testing can also be ensured when energy-stored surge is adopted to generate surge current.

Generally speaking, a wave form generated by a transformer is definitely a sine wave without any disturbance. Otherwise, the wave form may be just like the ones shown in Figure 3. 


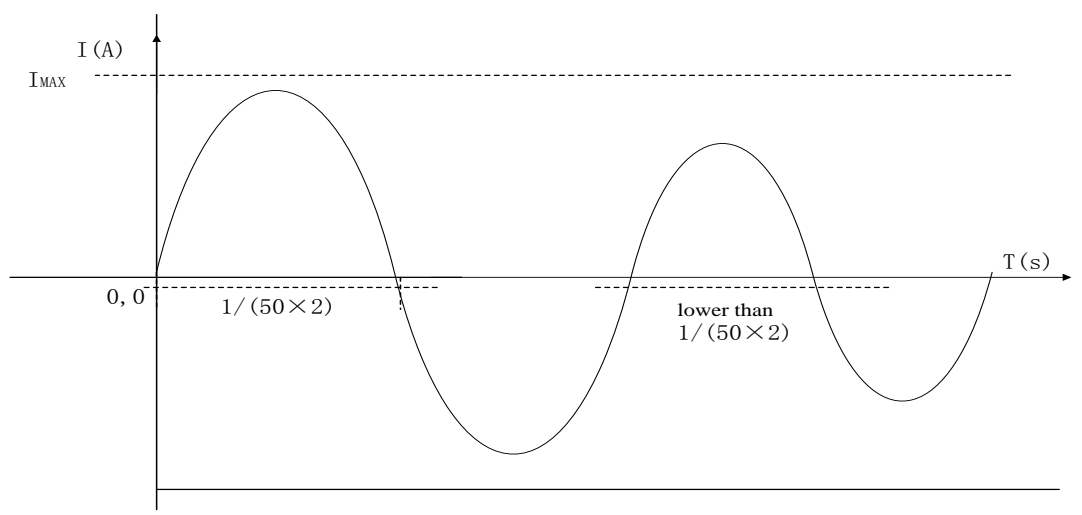

Figure 3. The waveforms generated by a transformer

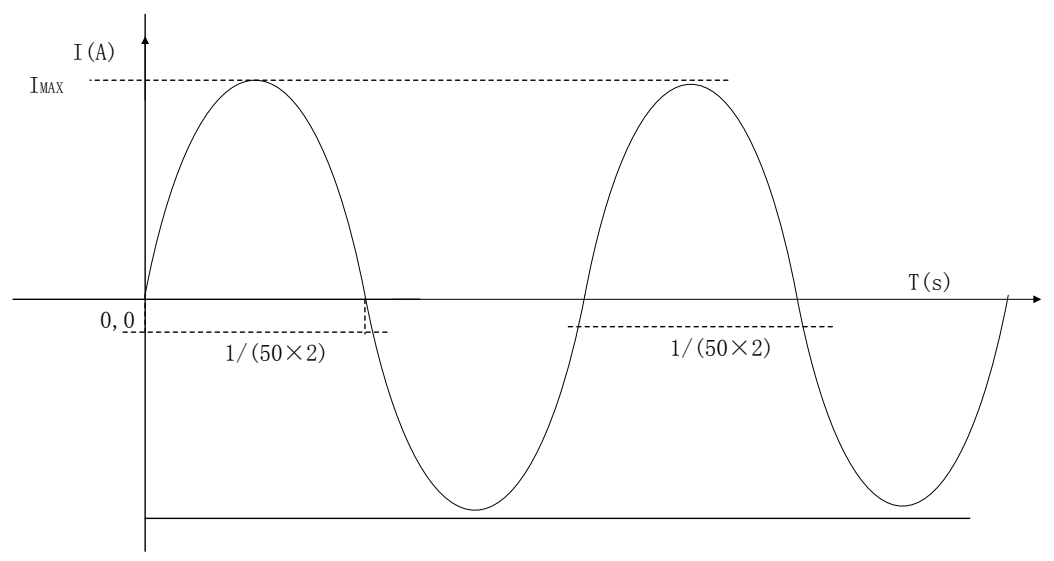

Figure 4. The waveforms generated by our method

The experiments show that the result can be highly improved and yield to a distinctive ladder-effect shown in Figure 4.

The wave shape showed in Figure 4 almost matches the ones generated only by numerical technologies. By adopting properly efficient filter capacitors used after voltage regulator to maintain a constant DC power supplies, the frequency of such sine wave is capable of being controlled and adjusted to $50 \mathrm{~Hz}$ with a minimum error.

One conclusion has to be arrived that such energy-stored surge current testing equipment has an immunity to the outside various kinds of disturbance and therefore fully meet the national testing standards.

(3) Comparison on power consumption and cost

Using transformer to generate 5.0kA surge current, higher power transformers are needed, which will make transformers bigger in volume, high loss, more materials and higher cost. Such disadvantages can be tackled when the equipments are produced by energy-stored surge current test.

\section{(4) Comparison on others}

Traditional transformer is unable to generate an inrush current with the peak of 5.0kA. Even if there are transformers meeting such requirements, other loads in the grid will be heavily impacted at the moment of surge current testing [5]. The instruments, developed with 
the method of energy-stored testing, will have nearly no impact on other loads in grid while charging and generating surge current.

\section{Conclusions}

This paper analyzes the importance of surge current generation and test system, and then a novel energy-stored surge current testing method is fully representation of the fundamental principles in small or medium-high scale circuit.

In the method presented above, the surge current is firstly generated by stored charges instead of the conventional transformers. Secondly, a proper microcomputer is employed to programmably and automatically control its surge waveform. Thirdly, the parameters, including the currents and voltages through the rectifier tube can be easily calculated and displayed in real time.

The contributions and disadvantages of our method are also briefly concluded. One of the full applications of basic charge and current principles has the incomparable advantages over those traditional surge current testing methods (e.g., transformer) in cost, power consumption and performance. In addition, it may compensate for other drawback of defects resulted from those surge current generated by transformer. More especially, it's proved experimentally to be much more accurate, controllable in the most important parameters such as surge time and peak.

However, there also exists some weakness of the method addressed above. For example, the capacitors charge and discharge times are limited as the capacitor is used to automatically complete charge storage in the designed circuits of the system [6]. Meanwhile, it evidently takes a long time for charging in the surge current testing period, which makes it restricted in the test application and thus should be improved in the further research. A key goal of this paper is to be helpful to some extent as a favorite reference for readers, researchers and designers in several engineering applications and the corresponding project design.

\section{Acknowledgements}

This work was supported by Jiangsu Intelligent Sensor Network Project Technology Research Centre Open Found (No.ZK12-04-05).

\section{References}

[1] The Chinese National Standard GB/4023-1997.

[2] L. Changbai and Z. Yinghao, "Calculation of power transformer", Heilongjiang science and technology press, Ha'erbin, (1990).

[3] BT65C Manual, Texas Instruments Incorporated, (1995).

[4] Department of Mathematics of TongJi University, "Advanced mathematics", Higher Education Press, Beijing (2006).

[5] W. Shaojie and L. An, "Development of harmonics and var compensation equipment for distribution grid with low-voltage high-current load", Electric power automation equipment, (2010), pp. 25-41.

[6] Z. Wanglong, "Selection and application of aluminum", Electrolytic capacitor electric welding machine, vol. 37, no. 5, (2007), pp. 35-38. 


\section{Authors}

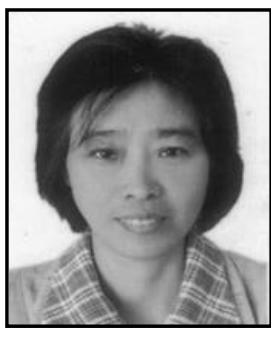

Dai Juan received her B. Eng degree in Optoelectronic Technology from Chengdu Institute of Telecommunication Engineering (e.i. University of Electronic Science and Technology of China) in 1984. Now she is currently researching on technology for "the Internet of things". She is an associate professor in Nanjing Institute of Industry Technology. Many of her projects supported by National foundation and Nanjing government have been accomplished by her and her team, which she played a leading part in it.

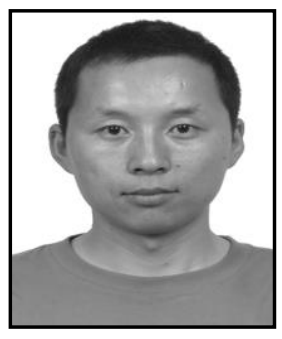

Zhao Zhihong graduated from Nanjing Financial University in 1997, and received his M. Eng degree in Mech-Electronic from Hohai Univeristy in 2004 and Ph.D Eng in Control Theory and Control Engineering in Donghua University in 2012. Now he is currently researching on technology for "the Internet of things" in Nanjing Institute of Industry Technology. He has been researching in computer graphic and image process for years and published over twenty professional papers in international journals and conferences. 